\title{
Sun protection using nanoparticles
}

\section{Alexey Popov}

Even after multiple applications of sunscreen, the UV-blocking particles remain localized close to the skin surface and produce insignificant numbers of free radicals.

With the depletion of the ozone layer due to human industrial activity on the one hand, and our increasing desire to stay outdoors during our holidays on the other, protection from UV radiation - in addition to that naturally provided by our skinis essential. This is, of course, the main purpose of sunscreen use. Protective properties of sunscreens work on the basis of absorption and scattering of incident radiation by their ingredients (UV filters). These chemical and physical compounds are organic molecules that absorb UV light and mineral nanoparticles that exhibit absorbing and scattering properties, respectively.

However, not all particle sizes are equally useful for UV protection. Both a good 'look' on the skin and adequate sunprotection properties are required. In addition, significant concerns as to the safety of nanoparticles have been raised in recent years. In particular, there are fears about particle penetrationafter multiple applications-through the superficial horny skin layer to living tissue. The formation of harmful free radicals in the presence of such particles under UV irradiation is another serious concern.

Studies simulating behavior of sunbathers on a beach during their holidays were carried out using volunteers. ${ }^{2}$ After multiple applications of sunscreens containing 100nm titaniumdioxide $\left(\mathrm{TiO}_{2}\right)$ nanoparticles, over a period of four days, stratum corneum (the horny layer) was removed by consecutive application of adhesive tape. Analysis of the tape containing skin cells using x-ray fluorescence spectroscopy revealed the $\mathrm{TiO}_{2}$ content of each strip and allowed reconstruction of an in-depth particle-penetration profile. The localization of the particles was predominantly superficial, within 1-3 microns from the surface. Other studies also show insignificant penetration of the skin by nanoparticles. ${ }^{3,4}$

The effect of particle size on UV-light attenuation was investigated on the basis of Mie scattering off spherical $\mathrm{TiO}_{2}$ nanoparti-

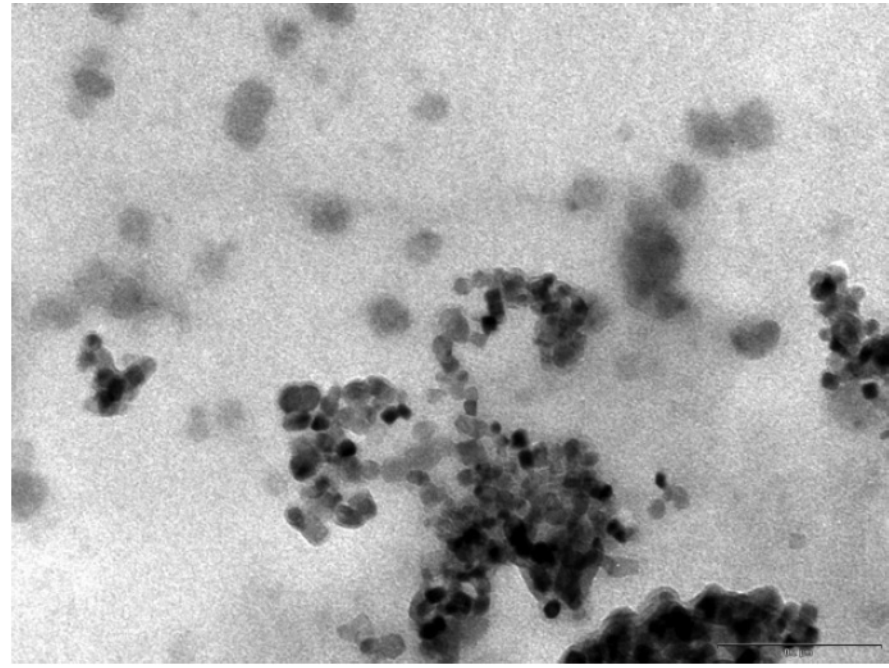

Figure 1. Transmission-electron-microscope image of $25 \mathrm{~nm} \quad \mathrm{TiO}_{2}$ nanoparticles in a diluted cream. ${ }^{1}$ Scale bar: $0.2 \mu \mathrm{m}$.

cles (rutile variety) and using a Monte-Carlo method for photon migration inside the stratum corneum. ${ }^{5}$ For increasingly shorter wavelengths, $\mathrm{TiO}_{2}$ particle sizes must be reduced to decrease the UV intensity. For example, for radiation at $310 \mathrm{~nm}$, the most appropriate size is $62 \mathrm{~nm}$, while for $400 \mathrm{~nm}$ light this increases to $122 \mathrm{~nm}$ particles. This difference is due to the mechanisms causing light attenuation: shorter-wavelength radiation is mainly absorbed by particles, while both absorption and reflection (although to a lesser extent) play a role at longer wavelengths.

We also investigated the formation of free radicals on glass slides and porcine skin (in vitro) in the presence of $\mathrm{TiO}_{2}$ nanoparticles (anatase variety) embedded in sunscreens. ${ }^{1}$ Particles of 25 and $400 \mathrm{~nm}$ diameter were used in the experiments (see Figure 1). We used electron paramagnetic-resonance spectroscopy to detect emerging free radicals. Smaller coated $\mathrm{TiO}_{2}$ nanoparticles were more photoactive than larger particles. This is clearly seen if the particles embedded in a placebo (i.e., sunscreen without UV filters) are applied on glass. Mie theory

Continued on next page 
explains the difference in light absorption by particles of a range of sizes and, as a consequence, differences in the amounts of free radicals generated. However, if the particles are applied on porcine skin, no distinct difference is observed. This is caused by the high skin contribution to free-radical production. Compared to the skin's ability to generate radicals, nanoparticles do not play a significant role in the concentrations used (sunscreen surface density: $2 \mathrm{mg} / \mathrm{cm}^{2}$ ).

In summary, our research-focusing on the effects of nanoparticle-skin interactions-shows that the mineral compound of sunscreens is (thus far) not an issue of concern. Even after multiple applications, the particles remain localized close to the surface and their radical-production ability does not surpass that of porcine skin in vitro. However, the latter effect could be more distinct in vivo: the applicability of our results to human skin must still be explored.

The author wishes to thank Jürgen Lademann, Alexander Priezzhev, and Risto Myllylä for fruitful discussions and assistance and the Academy of Finland for financial support.

\section{Author Information}

\section{Alexey Popov}

Optoelectronics and Measurement Techniques Laboratory University of Oulu

Oulu, Finland

Alexey Popov is a researcher with scientific interests in the area of nanoparticles used in sunscreens. After having obtained his $\mathrm{PhD}$ from M. V. Lomonosov Moscow State University (Russia) in 2006, he continued his research in Finland, leading to a DSc (Tech.) degree in 2008. He has published over 60 papers and coorganized an international conference on advanced laser technologies (ALT-2007), held in Finland.

\section{References}

1. A. P. Popov, S. Haag, M. Meinke, J. Lademann, A. Priezzhev, and R. Myllylä, Effect of size of $\mathrm{TiO}_{2}$ nanoparticles applied to glass slide and porcine skin on generation of free radicals under ultraviolet irradiation, J. Biomed. Opt. 14 (2), 2009. In press (March).

2. J. Lademann, H.-J. Weigmann, C. Rickmeyer, H. Barthelmes, H. Schaefer, G. Mueller, and W. Sterry, Penetration of titanium dioxide microparticles in a sunscreen formulation into the horny layer and the follicular orifice, Skin Pharmacol. Appl. 12, pp. 247-256, 1999. doi:10.1159/000066249

3. G. J. Nohynek, J. Lademann, C. Ribaud, and M. Roberts, Grey goo on the skin? Nanotechnology, cosmetic and sunscreen safety, Crit. Rev. Toxicol. 37, pp. 251-277, 2007. doi: $10.1080 / 10408440601177780$

4. A. V. Zvyagin, X. Zhao, A. Gierden, W. Sanchez, J. A. Ross, and M. S. Roberts, Imaging of zinc oxide nanoparticle penetration in human skin in vitro and in vivo, J. Biomed. Opt. 13 (6), p. 0640311, 2008. doi:10.1117/1.3041492

5. A. P. Popov, J. Lademann, A. V. Priezzhev, and R. Myllylä, Effect of size of $\mathrm{TiO}_{2}$ nanoparticles embedded into stratum corneum on ultraviolet- $A$ and ultraviolet$B$ sun-blocking properties of the skin, J. Biomed. Opt. 10 (6), p. 0640371, 2005 doi:10.1117/1.2138017 